ACCEPTED FOR PUBLiCATION IN APJL

Preprint typeset using $\mathrm{LAT}_{\mathrm{E}} \mathrm{X}$ style emulateapj v. 04/20/08

\title{
HYSTERESIS OF BACKFLOW IMPRINTED IN COLLIMATED JETS
}

\author{
Akira Mizuta $^{1}$, Motoki Kino ${ }^{2}$, And Hiroki Nagakura ${ }^{3}$ \\ accepted for publication in ApJL
}

\begin{abstract}
We report two different types of backflow from jets by performing $2 \mathrm{D}$ special relativistic hydrodynamical simulations. One is anti-parallel and quasi-straight to the main jet (quasi-straight backflow), and the other is bent path of the backflow (bent backflow). We find that the former appears when the head advance speed is comparable to or higher than the local sound speed at the hotspot while the latter appears when the head advance speed is slower than the sound speed at the hotspot. Bent backflow collides with the unshocked jet and laterally squeezes the jet. At the same time, a pair of new oblique shocks are formed at the tip of the jet and new bent fast backflows are generated via these oblique shocks. The hysteresis of backflow collisions is thus imprinted in the jet as a node and anti-node structure. This process also promotes broadening of the jet cross sectional area and it also causes a decrease in the head advance velocity. This hydrodynamic process may be tested by observations of compact young jets.
\end{abstract}

Subject headings: hydrodynamics - galaxies: jets - methods: numerical - relativity

\section{INTRODUCTION}

Recent observations of jets in active galactic nuclei (AGN) clearly tell us their morphological characters. On VLBI scales, the MOJAVE (Monitoring Of Jets in Active galactic nuclei with VLBA Experiments) program clearly shows us a number of actual internal inhomogeneous structures, and a growth of the jet's cross-sectional area along its axis (http://www.physics.purdue.edu/ mlister/MOJAVE/index Lister et al. (2009)). 'Their cross sections are not constant nor internally uniform. The importance of jet's internal structures has also been used to explain multi frequency spectra Ghisellini et al. 2005). From the standpoint of hydrodynamics, however, the generation of internal structures in jets and what determines the cross sectional area of jets during their evolutions have not yet been fully investigated.

Most prior work has focused on Kelvin-Helmholtz instability (KHI) caused by the velocity shear between a jet and surrounding matter (e.g., Hardee \& Norman (1988); Perucho et al. (2006)). The instability is known as a trigger of diamond-shape internal structures caused by oblique shocks inside the unshocked jet (e.g, Norman et al. 1984). Once the unshocked jet is perturbed by the KHI in a cocoon which surrounds the jet (Begelman et al. 1984), then an oblique shock appears inside the unshocked jet and the velocity component perpendicular to the jet axis is canceled out through this oblique shock (Komissarov \& Falle 1997, 1998).

In this Letter, we newly show the importance of backflow. A backflow is usually generated at the hotspot of the light jet, where a light jet is a jet with mass density $\left(\rho_{\mathrm{j}}\right)$ lower than that of the ambient gas $\left(\rho_{\mathrm{a}}\right)$, where the subscripts ' $j$ ' and ' $a$ ' stand for the jet and ambient

\footnotetext{
${ }^{1}$ Center for Frontier Science, Chiba University Yayoi-cho 1-33, Inage-ku, Chiba, 263-8522, Japan

2 National Astronomical Observatory of Japan, Mitaka, 1818588, Japan

${ }^{3}$ Science and Engineering, Waseda University, 3-4-1 Okubo, Shinjuku, Tokyo 169-8555, Japan
}

gas, respectively. Although the existence of backflows is well known to previous studies by numerical simulations of the jet propagation (Norman et al. 1982; Marti et al. 1997; Rosen et al. 1999; Aloy et al. 2000; Zhang et al. $2003)$, the importance of the backflow in terms of dynamics is not well recognized. The backflow dynamics are usually ignored since the backflow is subsonic flow. For the first time, it is shown that backflow is essential fir forming the internal structure and the growth rate of the cross-sectional area of jets.

\section{NUMERICAL SET-UP}

We utilize the axisymmetric two dimensional special relativistic hydrodynamic code in cylindrical coordinate $(r \times z)$ developed by one of the authors (AM: see for details, Mizuta et al. (2004, 2006)). Using the PPM method and TVD Runge-Kutta time integration, we achieve 3rd order accuracy in terms of space and time. Since we do not include any radiative process, the equations solved in this study are scale free. The ideal gas equation of state $(p=(\gamma-1) \rho \epsilon)$ is adopted, where $p$ is pressure, $\epsilon$ is specific internal energy, and $\gamma$ is specific heat ratio which is fixed to $5 / 3$. The local sound speed is derived as $c_{s}=\sqrt{\gamma p / \rho h}=\sqrt{\epsilon \gamma(\gamma-1) /(1+\epsilon \gamma)}$.

The boundary condition at the cylindrical axis $(r=$ 0 ) is the reflective condition. The reflective boundary condition is assumed at $z=0$ except for the first 10 grid points, i.e., $0<r<1 R_{b}$ where a continuous jet is imposed. The $R_{b}$ is the radius of the injected jet from the computational boundary $(z=0)$. A zero gradient free boundary condition is assumed at $z=100 R_{b}$ and $r=r_{\max }$. The unit of the space scale is the beam radius of the injected jet $\left(R_{b}\right)$. The unit of the time scale is $R_{b} / c$, where $c$ is speed of light.

In this study, five cases with different jet velocities $\left(v_{j}\right)$ have been examined these are $v_{j}=0.9 c$ (model J09), $v_{j}=0.8 c(\mathrm{~J} 08), v_{j}=0.7 c(\mathrm{~J} 07), v_{j}=0.6 c(\mathrm{~J} 06)$, and $v_{j}=0.5 c(\mathrm{~J} 05)$. Corresponding Lorentz factors $(\Gamma)$ are 2.3 (J09), 1.7 (J08), 1.4 (J07), 1.3 (J06) and 1.2 (J05), respectively. The jet is constantly injected from one of the computational boundaries. Other model parameters 
are fixed as follows. The density ratio is fixed to be $\eta \equiv \rho_{j} / \rho_{a}=0.1$. The initial mass density profile of the ambient matter $\rho_{a}$ is assumed to be uniform. Mass densities are normalized by the ambient gas, i.e., $\rho_{a}=1$. Both the jet and the ambient gas are assumed to be cold, i.e., $\epsilon_{j} / c^{2}=10^{-2}$ and $\epsilon_{a} / c^{2}=10^{-6}$, where $\epsilon$ is specific internal energy. Thus specific enthalpy $\left(h \equiv 1+\epsilon / c^{2}+\right.$ $p / \rho)$ is $\sim 1$. Corresponding sound speeds of the jet and ambient gas are $c_{s, j} \sim 0.16 c$ and $c_{s, a} \sim 1.6 \times 10^{-3} c$, respectively. The velocity vector of the injected jet is parallel to the cylindrical axis. We intend to do extensive studies with wider parameter space in the near future.

The numerical runs are performed until the head of the jet reaches the boundaries at $z=100 R_{b}$. Since 10 uniform grid points per a $R_{b}$ are given in all computational domains for both radial and $z$ directions, there are in total 1000 grid points in the $z$ direction. Since the aspect ratio of the distances between the propagation direction and lateral direction is different in each model, we set different computational domains $\left(r_{\max }\right)$ in $r$ by the models so that the sideways expanding shock is inside the computational domain in the last stage of the computation.

\section{NUMERICAL RESULTS}

Here we mainly discuss the results of J09 and J05 in order to study differences in backflow dynamics more clearly. The other three cases will be briefly summarized later on. Figure 1 shows the mass density contours at the final stage of each model (model J05: $t=1845 R_{b} / c$, model J09: $\left.t=255 R_{b} / c\right)$. Significant differences are found between the two models. They are characterized as follows.

(P1) Significant nodes/anti-nodes structure is generated along the jet in J05. The cross sectional radius varies along the jet in J05, while the cross sectional radius is almost constant along the jet in J09.

(P2) The radii of the jet at the terminal shock are about $1.5 R_{b}$ in J09, whilst the radii is $5 R_{b}$ in $\mathrm{J} 05$ which is wider than that in J09.

(P3) The aspect ratio of the surrounding bow shock appearing in J09 is narrower than the one in J05.

In both models, we observe the backflow which begins the hotspot at the head of the jet. The temperature in the hotspot is quite high, since all the jet's kinetic energy is converted to thermal energy through the terminal shock. The local sound speeds in the hotspot are $c_{\mathrm{s}, \mathrm{hs}} \sim 0.5 c$ (model J09) and $c_{\mathrm{s}, \mathrm{hs}} \sim 0.3 c$ (model J05). In order to clarify the physical reasons for these differences in the density map, below we examine the time evolution of each model in detail. In advance, we stress that the velocity field maps are the key to understanding the above difference because backflows play an important role in these maps.

Figure 2(a) shows the contour of the absolute velocity (color) and velocity vectors (arrows) of the model J09 in its early phase (at $t=30 R_{b} / c$ ). The jet (red region in contour in Fig $2(\mathrm{a})$ ) ends at the terminal shock (at $z=13 R_{b}$ ), forming a hotspot. We find that the jet is surrounded by a layer which is made of shocked matter escaped from the hotspot. The velocity of this flow is quite small and it sometimes shows a negative value measured from the observer frame, i.e., $v_{z}<0$. This is because $c_{\mathrm{s}, \mathrm{hs}}$ and the head propagation speed $v_{h} \sim\left(14 R_{b}\right) /\left(30 R_{b} / c\right) \sim 0.5 c$ are very similar, where $v_{h}$ is the speed of the contact discontinuity between the shocked jet and the shocked ambient gas along the jet axis. The path of this narrow flow is also almost straight near the head (quasi-straight backflow) in the late phase of evolution, (see Fig. 2(b)). Since the straight backflow is unstable for the KHI between the jet and the backflow, some vortices are generated along the jet.

Figures 2(c)-(f) show the evolution of the absolute velocity contours for model J05. It is found that the path of the backflow of model J05 differs dramatically from that of model J09. At $t=105 R_{b} / c, v_{h} \sim\left(13 R_{b}\right) /\left(105 R_{b} / c\right) \sim$ $0.1 c$. The path of the backflow from the hotspot is not straight but bent (bent backflow) near the head, (see Fig. 2(d)). The speed of the bent backflow near the hotspot is $\sim 0.25 c$. This bent backflow compresses and piles up the shocked ambient gas. Then, the backflow makes a half loop path. Finally, the bent backflow vertically collides with the main jet from the side, (see Fig. $2(\mathrm{~d})$ at $\left.z=7 R_{b}\right)$.

As shown above, the transition of backflow dynamics is caused by different head advance velocity. The head advance velocity decreases from model J09 to model J05. The intermediate models (J08, J07) show quasi-straight backflow paths, while the backflow of the model J06 exhibits bent path when each head reaches $z \approx 12 R_{b}$, see Fig 3 .

\section{PHYSICAL INTERPRETATIONS}

In this section, we explain the physical reasons for the significant difference between the two models. According to our simulations, the backflow dynamics are the most important feature in understanding these differences and we have found mainly two features. One is the difference in the ratio of $v_{\mathrm{h}}$ to $c_{\mathrm{s}, \mathrm{hs}}$. As we have shown, $v_{\mathrm{h}}$ is comparable to $c_{\mathrm{s}, \mathrm{hs}}$ in model J09, whereas the head propagation speed is slower than local sound speed in model J05. Since the backflow starts the hotspot, the speed of the backflow at the frame of the hotspot is close to $c_{\mathrm{s}, \mathrm{hs}}$. If $v_{\mathrm{h}}$ is slower than the local sound speed, the backflow expands sideways. In the other case, i.e. model J09, the backflow goes directly backward and flows anti-parallel flow to the main jet, see a schematic figure of the two types of the backflow path (top panel of Fig. 4). By the comparison of Fig. 2(a) and Fig. 2(d), it is found that the jets propagated at the same distance from the two models but along different paths of the backflow, i.e., quasistraight backflow and bent backflow. The bent backflow is generated when the condition $c_{\mathrm{s}, \mathrm{hs}} / v_{\mathrm{h}}<1-2$ holds. For $c_{\mathrm{s}, \mathrm{hs}} / v_{\mathrm{h}} \sim 2$, the backflow is clearly separated from the jet.

The other reason is the emergence of the oblique shock at the tip of the jet-terminal which is shown in Fig. 4 (the red line in the top panel). The bottom panel of Fig 4 shows log-scaled density (top) and pressure (bottom) contours of the same model and at the same time of Fig. 2 (e), i.e., model J05 at $t=270 R_{b} / c$. The density and pressure discontinuity can be clearly seen at $15<z / R_{b}<20,|r| \sim 1 R_{b}$. This is an oblique shock and is indicated by the dashed line in the contours (Fig. 4), see also Fig. 8(b) (Mizuta et al. 2004) in 
which the oblique shock and fast backflow emerging thorough the shock can be clearly seen. When the oblique shock appears, the backflow comes out not only from the hotspot but also from the oblique shock, as shown in the shematic figure in Fig 4 A weakly dissipated flow passes through such an oblique shock and it becomes bent backflow. The speed of the bent backflow is $\sim 0.5 c$. Although little attention has been paid to such a weakly dissipated flow, it is essential for producing backflows. This weakly dissipated flow was seen in some previous studies i.e., Saxton et al. (2002); Mizuta et al. (2004). Since this flow does not pass through hotspots, the flow directly becomes a backflow, keeping its high velocity. This flow is separated from the main jet and draws a half loop in the cocoon. As a result, a new oblique shock emerges near the terminal, when the bent backflow vertically hits the unshocked main jet.

P1 and P2 are understood as follows. Above, we elucidate that the ratio $v_{\mathrm{h}} / c_{\mathrm{S}}$,hs divides the types of backflows. Below, we explain how P1 and P2 are achieved. When the bent backflow laterally collides with the unshocked jet, the jet is squeezed by the dynamical pinch. Thus oblique shocks are generated inside the jet. Just ahead of the squeezed node, the jet starts to expand like a nozzle. This process for generating oblique shocks is completely different from the previously known one in which internal shocks are caused by a pressure fluctuation in the cocoon (e.g., Norman et al. 1982). Since the terminal shock is close to the collisional point, this oblique shock does not work very well to reconfine the jet. On the contrary, the cross section of the jet increases at its terminal as shown in the schematic figure (Fig. 4). The wider the cross section becomes, the slower the jet advance speed becomes. Furthermore, a pair of oblique shocks represented by the red line reaches the terminal of the jet and connects with the reverse shock. Once the bent backflow appears from the hotspot, it is easy to generate these oblique shocks, and new bent backflows intermittently emerge from them as discussed above. The resultant structure therefore has significant node/anti-node structures which are the hysteresis of the backflow attacks as pointed out in P1 and P2.

In J09, $v_{\mathrm{h}}$ is almost constant throughout the whole calculation. In J05 $v_{\mathrm{h}}$ is also constant up to $t \sim 200 R_{b} / c$. After that, the head propagation velocity begins to decelerate as is shown in the numerical studies of Scheck et al. (2002) and Mizuta et al. (2004). A large vortex is formed near the hotspot in the simulations. The large vortex causes the deceleration of the head propagation. Kawakatu \& Kino (2006) analytically obtain $v_{\text {h }}$ taking the growth of head cross sectional area into account and it reproduces these numerical results well. Thus, P3 can be simply understood in terms of previous studies, i.e., the deceleration of $v_{\mathrm{h}}$. Finally, it should be noted that the bent backflow is indirectly linked with the generation for a large vortex, since a weak dissipated flow through an oblique shock is a key for the appearance of the vortex (Mizuta et al. 2004).

So far, we fix $\gamma=5 / 3$ for simplicity. This is inappropriate when the specific internal energy is in a relativistic regime $\left(\epsilon / c^{2} \gtrsim 1\right)$, and it could be achieved in the hotspot. Since the specific heat ratio should be smaller than 5/3 in the relativistic regime, the actual backflow velocity from the hotspot is slightly slower than our es- timation. Therefore, the transition velocity of backflow dynamics is also smaller. In order to check the validity of our discussions thus far in the real system, we examine the case of fixed $\gamma=4 / 3$ which corresponds to the relativistic limit. Three injection velocities, $v_{j}=0.4 c$ (model J04-43), $v_{j}=0.5 c$ (model J05-43), and $v_{j}=0.9 c$ (model J09-43) are studied. Figure 5(a)-(c) show the absolute velocity of these models in the early phase of the evolution. These simulations also show that there are two types of backflows, the quasi-straight path (models J05-43 and J09-43) and bent path (models J04-43). It is verified that the transition velocities are $v_{j} \sim 0.4 c$, whereas $v_{j} \sim 0.6 c$ in the fixed $\gamma=5 / 3$ case. That is, the true transition would be a certain value between them. Therefore we conclude that our simplification does not change the essence of our new findings.

\section{SUMMARY AND DISCUSSION}

In this work, we show two types of backflow dynamics, quasi-straight path and bent path. The difference can be clearly seen in the early phase of the dynamics, (see Figs. 2 (a) and (c)). When the head propagation velocity is smaller than local sound speed at the hotspot, a bent backflow appears. The bent backflow goes backward and sometimes attacks the jet from the side. When the jet is dynamically attacked by the bent backflow, the cross section of the jet increases, making an oblique shock. At the terminal of the jet, an oblique shock and the terminal reverse shock connects, making a weak dissipated flow into the cocoon through the oblique shock. Such backflow also becomes a bent one and appears repeatedly. The transition of two types of backflow dynamics occurs by decreasing the head propagation velocity.

Compact Symmetric Object (CSO) would be one of the best targets for testing our findings, since CSOs are newly born AGN jets with radio lobes. For instance, one of the smallest CSO radio lobes 3C 84 Asada et al. (2006) with the size of $\sim 10 \mathrm{pc}$ may be a good target to explore the backflow physics investigated in this work. Observations with unprecedented high spatial resolution which will be provided by VLBI Space Observatory Programme 2 (VSOP-2) in the near future appear to provide a promising means of exploring these backflows.

We add brief comments on the relevance to gamma-ray bursts (GRBs). Hot jets are expected in GRBs and it is intriguing to see how backflows act when jets are hot. Since the GRB jet from collapsars is formed deep inside of the progenitor, the jet should drill the high density stellar envelopes to reach the progenitor surface (e.g., Lazzati et al. (2009)). For example, the bent path of the backflow from the head of the GRB jet is indeed seen in Figures 2, 3 and 4 in Zhang et al. (2004), during its propagation in the high density progenitor envelope.

We are grateful to an anonymous referee for beneficial comments. This work is partly supported by the Grantsin-Aid of the Ministry of Education, Science, Culture, and Sport (20041002, 19540236, 21018002 A.M.). This work was carried out on NEC SX8, at Yukawa Institute for Theoretical Physics, Kyoto University, on the Space Science Simulator (NEC SX6), at the Japan Aerospace Exploration Agency, and on XT4 system and NEX SX9, at the Center for Computational Astrophysics, National 
Astronomical Observatory of Japan.

\section{REFERENCES}

Aloy, M. A., Müller, E., Ibáñez, J. M., Martí, J. M., \& MacFadyen, A. 2000, ApJ, 531, L119

Asada, K., Kameno, S., Shen, Z.-Q., Horiuchi, S., Gabuzda, D. C., \& Inoue, M. 2006, PASJ, 58, 261

Begelman, M. C., Blandford, R. D., \& Rees, M. J. 1984, Reviews of Modern Physics, 56, 255

Ghisellini, G., Tavecchio, F., \& Chiaberge, M. 2005, A\&A, 432, 401

Hardee, P. E., \& Norman, M. L. 1988, ApJ, 334, 70

Kawakatu, N., \& Kino, M. 2006, MNRAS, 370, 1513

Komissarov, S. S. \& Falle, S. A. E. G. 1997, MNRAS, 288, 833

Komissarov, S. S., \& Falle, S. A. E. G. 1998, MNRAS, 297, 1087

Lazzati, D., Morsony, B. J., \& Begelman, M. C. 2009, ApJ, 700, L47

Lister, M. L., et al. 2009, AJ, 137, 3718

Marti, J. M. A., Müller, E., Font, J. A., Ibanez, J. M. A., \& Marquina, A. 1997, ApJ, 479, 151

Mizuta, A., Yamada, S., \& Takabe, H., 2004, ApJ, 606, 804

Mizuta, A., Yamasaki, T., Nagataki, S., \& Mineshige, S. 2006, ApJ, 651, 960

Mizuta, A., \& Aloy, M. A. 2009, ApJ, 699, 1261

Norman, M. L., Winkler, K.-H. A., Smarr, L., \& Smith, M. D. 1982, A\&A, 113, 285

Perucho, M., Lobanov, A. P., Martí, J.-M., \& Hardee, P. E. 2006, A\&A, 456, 493

Rosen, A., Hughes, P. A., Duncan, G. C., \& Hardee, P. E. 1999, ApJ, 516, 729

Saxton, C. J., Sutherland, R. S., Bicknell, G. V., Blanchet, G. F., \& Wagner, S. J. 2002, A\&A, 393, 765

Scheck, L., Aloy, M. A., Martí, J. M., Gómez, J. L., Müller, E. 2002, MNRAS, 331, 615

Zhang, W., Woosley, S. E., \& MacFadyen, A. I. 2003, ApJ, 586, 356

Zhang, W., Woosley, S. E., \& Heger, A. 2004, ApJ, 608, 365 

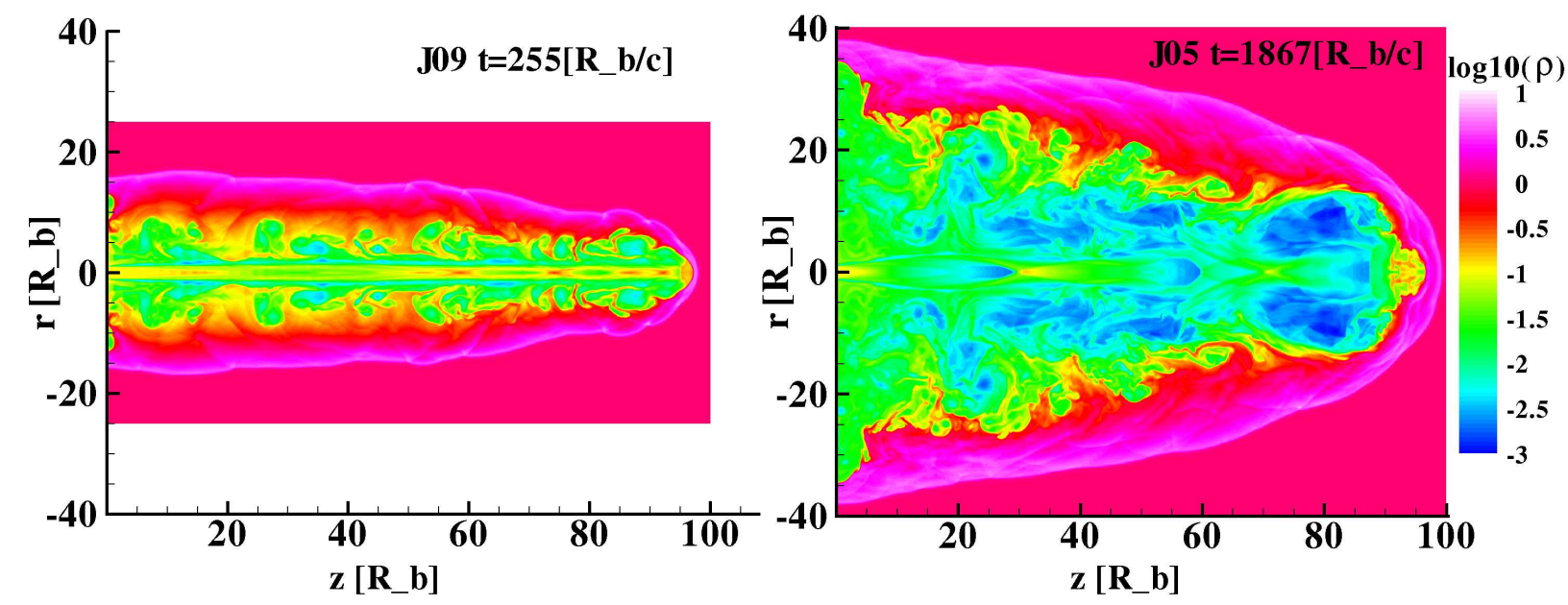

FiG. 1.- Density contours of the models J05 (left) and J09 (right) at the end of simulations. In both cases internal structures can be seen inside the jet. The jet radius increases at anti-node and decreases at the node for the case J05, while the radius of the jet is almost constant along the jet for the case J09. 

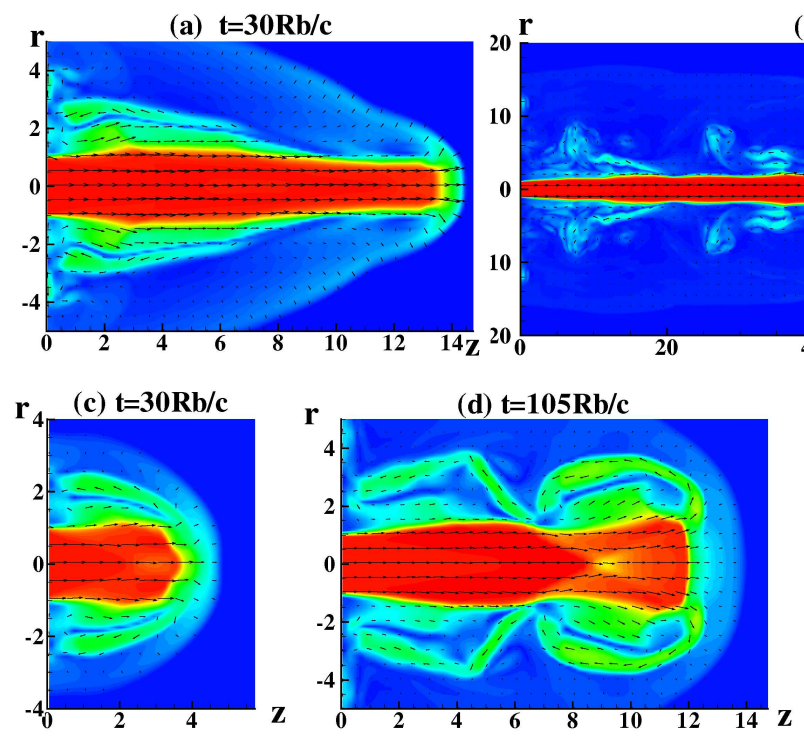

(b) $\mathbf{t}=255 \mathrm{Rb} / \mathrm{c}$
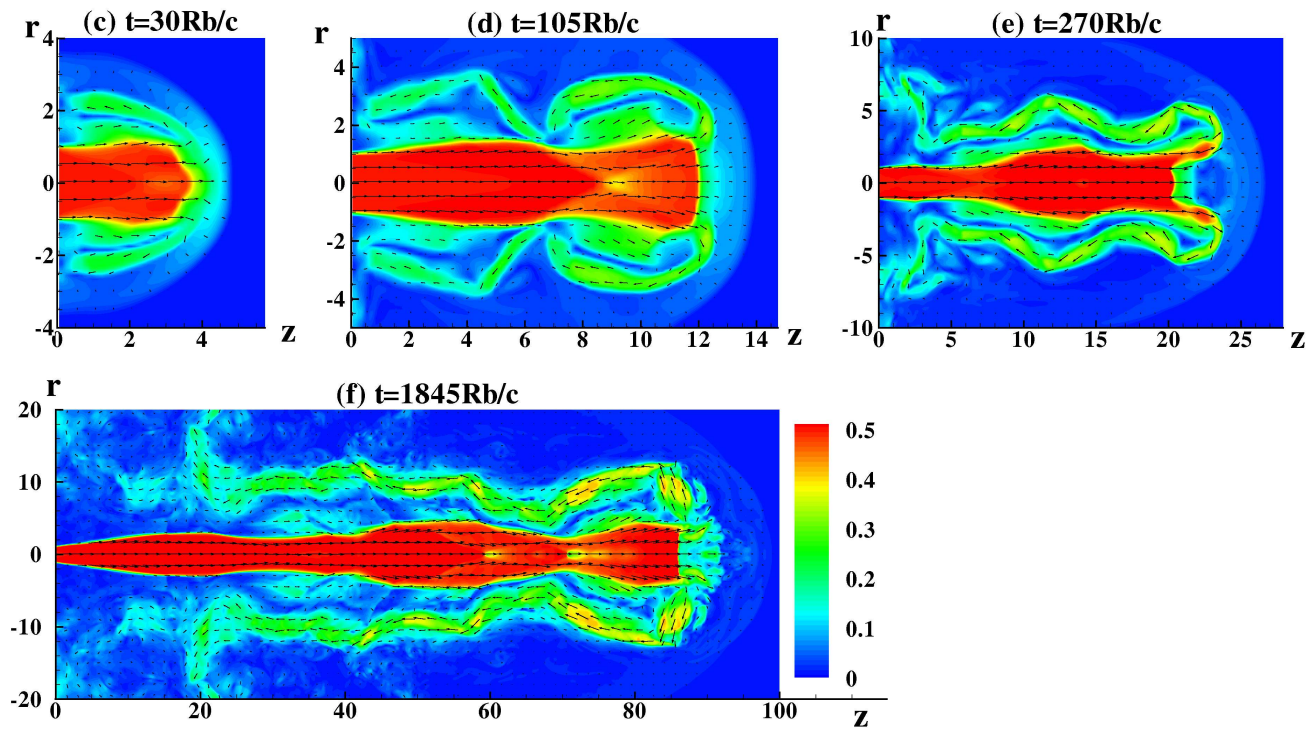

FIG. 2.- Absolute velocity contours of the model J09 at (a) $t=30$ and (b) $255 R_{b}$, and of the model J05 at (c) $t=30$, (d) 105, (e) 270, and (f) $1845 R_{b}$. The arrows show the velocity vectors. The color bars are common for (a) and (b), and for (c)-(f). The backflow is almost straight for the model J09. Whereas the backflow is bent for the model J05. A bent backflow attacks the jet from the side (d)-(f). 

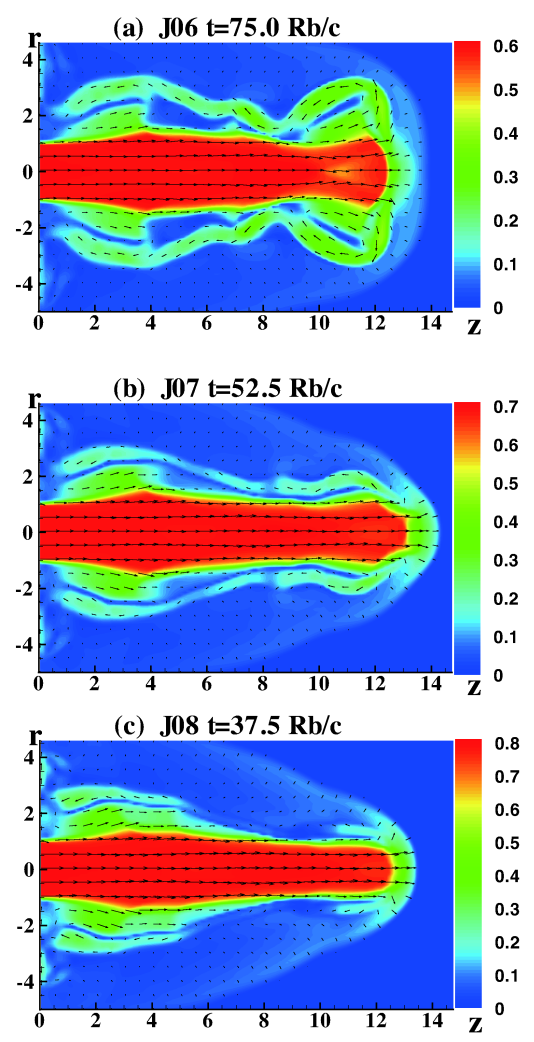

FIG. 3.- Same as Fig. 2 but for models (a)J06 $\left(t=75 R_{b} / c\right)$, (b)J07 $\left(t=52.5 R_{b} / c\right)$, and (c)J08 $\left(t=37.5 R_{b} / c\right)$. The jet propagates about $12 R_{b}$ in each models, see also Fig.2(a) and (d) for the comparison. The path of the backflow is quasi-straight for models J07 and J08. On the contrary the path of the backflow is bent for model J06. 


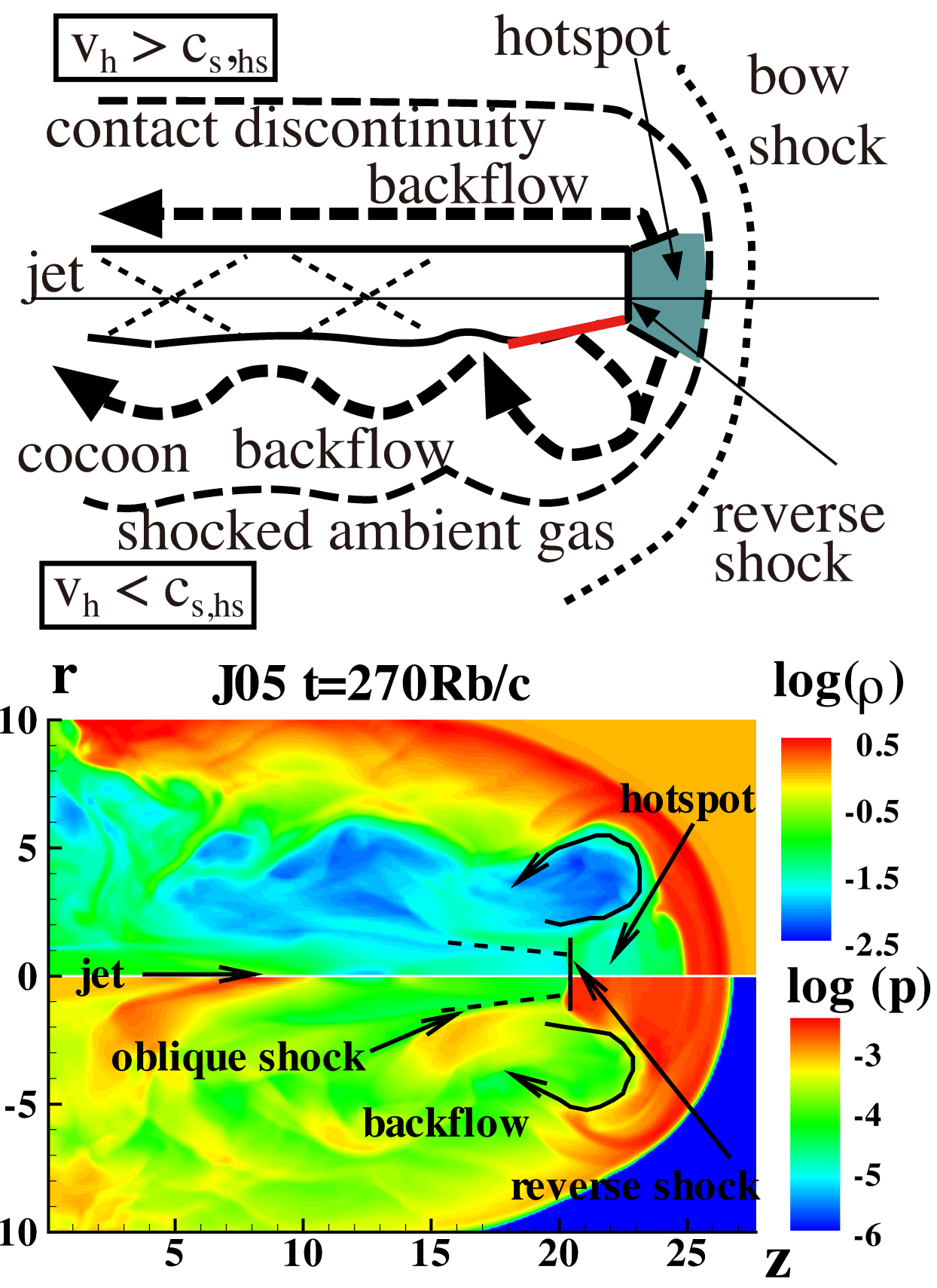

FIG. 4.- A schematic figure of the path of the jet and backflow (top). Two cases are shown. The head speed is faster than the local sound speed at the hotspot (upper top) and the head speed is slower than the local sound speed at the hotspot (lower half). The path of the backflow is quasi-straight for the former case. The path of the backflow is bent for the latter case. The backflow from the hotspot thermally expands sideways, and becomes bent backflow, interacting with the shocked ambient gas. An oblique shock sometimes appears (red line) at the terminal of the jet which is another way to trigger bent backflow. The bottom panel shows log-scaled density and pressure contours of the same model and at the same time of Fig. 2 (e), i.e., model J05 at $t=270 R_{b}$. Near the terminal shock which is at $z=20 R_{b}$ and perpendicular to the $z$ axis, a discontinuity indicated by the dashed line can be seen. This corresponds to an oblique shock shown by the red line in the top panel. The backflow mainly comes through this oblique shock at this snapshot, see also Fig. 2 (e) in which a fast backflow starts through this oblique shock and it becomes a bent backflow. 

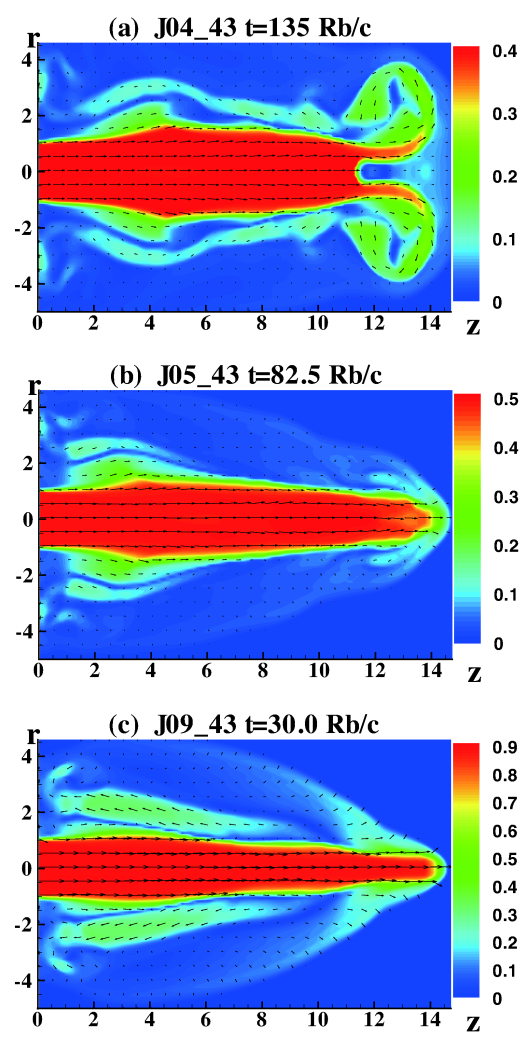

FIG. 5. - Same as Fig. 2 but for models (a) J04-43 $\left(t=127.5 R_{b} / c\right)$, (b) J05-43 $\left(t=82.5 R_{b} / c\right)$, and (c) J09-43 $\left(t=30 R_{b} / c\right)$. These simulations are done with fixed specific ratio $\gamma=4 / 3$. The path of the backflow is quasi-straight one (model J05-43 and J09-43). On the contrary the path of the backflow is bent one for model J04-43. The transition of backflow property occurs at slower injection velocity than that for the models done with fixed specific heat ratio $\gamma=5 / 3$. 